\title{
Distribución geográfica del sapito de rayas o sapo variegado Nannophryne variegata Günther, 1870, nuevas localidades y comentarios sobre su hábitat en Chile y Argentina
}

\section{Geographic distribution of the striped or variegated toad Nannophryne variegata Günther, 1870, new localities and comments on its habitat in Chile and Argentina}

\author{
Ingrid Cisternas-Medina ${ }^{1,2}$, Juan Carlos Ortiz ${ }^{1 *}$, Carmen Úbeda $^{3}$, Helen Díaz-PÁez $^{4} \&$ Marcela Vidal $^{5}$ \\ ${ }^{1}$ Laboratorio de Sistemática y Conservación de Herpetozoos, Departamento de Zoología, Facultad de Ciencias Naturales y \\ Oceanográficas, Universidad de Concepción, Concepción, Chile. \\ ${ }^{2}$ Programa Doctorado en Sistemática y Biodiversidad, Departamento de Zoología, Facultad de Ciencias Naturales y \\ Oceanográficas, Universidad de Concepción, Concepción, Chile. \\ ${ }^{3}$ Universidad Nacional del Comahue, Centro Regional Bariloche. Quintral 1250, 8400, San Carlos de Bariloche, Argentina. \\ ${ }^{4}$ Departamento de Ciencias Básicas, Escuela de Educación, Campus Los Ángeles, Universidad de Concepción, Los Ángeles, \\ Chile. \\ ${ }^{5}$ Laboratorio de Ecología y Evolución, Departamento de Ciencias Básicas, Facultad de Ciencias, Universidad del Bío-Bío. \\ Chillán, Chile \\ *jortiz@udec.cl
}

\begin{abstract}
RESUMEN
Nannophryne variegata es una especie endémica de los bosques australes de Chile y Argentina. Posee una amplia distribución en territorio chileno y en menor medida, en territorio argentino. A lo largo de esta extensa franja latitudinal de distribución, habita diferentes regiones ecológicas, caracterizadas principalmente por temperaturas bajas y alta precipitación. Escasos son los antecedentes respecto a las preferencias de hábitat y amenazas para esta especie. Este trabajo informa sobre nuevos registros de distribución en el extremo sur de Chile y Argentina. Describe, además, las características de los hábitats que ocupa y la representación en áreas protegidas de ambos países. Nannophryne variegata se distribuye desde $\operatorname{los} 37^{\circ} 28^{\prime}$ hasta los $52^{\circ} 10^{\prime}$ latitud sur, siendo uno de los anfibios más australes del mundo. Habita diferentes ambientes que comprenden asociaciones boscosas húmedas, turberas, tundra magallánica y ambientes altoandinos vecinos. Se encuentra bien representada en áreas protegidas con un gran número de poblaciones observadas.
\end{abstract}

Palabras Clave: conservación, distribución, hábitat, Nannophryne variegata.

\begin{abstract}
Nannophryne variegata is an endemic species of the southern forests of Chile and Argentina. It has a wide distribution in Chilean territory and to a lesser extent, in Argentine territory. Along this extensive latitudinal distribution, it inhabits different ecological regions, characterized mainly by low temperatures and high precipitation. There are few antecedents regarding habitat preferences and threats to this species. This paper reports on new distribution records in the extreme south of Chile and Argentina. It also describes the characteristics of the habitats it occupies and the representation in protected areas of both countries. Nannophryne variegata is distributed from $37^{\circ} 28^{\prime}$ to $52^{\circ} 10^{\prime}$ South latitude, becoming one the southernmost amphibians in the world. It inhabits different environments that include humid forest associations, peat bogs, Magellanic tundra and neighboring high Andean environments. It is well represented in protected areas with a large number of observed populations.
\end{abstract}

KEYWORDS: conservation, distribution, habitat, Nannophryne variegata.

\section{INTRODUCCIÓN}

Los bosques templados de Sudamérica, restringidos al sur de Chile $\left(37^{\circ}-55^{\circ} \mathrm{S}\right)$ y sectores adyacentes de Argentina, se caracterizan por tener pocas especies de anuros (Cei 1962). Formas (1979) señaló que la zona con menor concentración de anfibios se encuentra al sur del paralelo $50^{\circ} \mathrm{S}$, llegando a esta zona solamente los anuros del género Bufo (refiriéndose a Nannophryne). Sin embargo, actualmente se conocen cuatro especies (Batrachyla antartandica, Chaltenobatrachus grandisonae, Nannophryne variegata y Pleurodema bufoninum), presentes en bosques y turberas al 
sur de dicho paralelo (Atalah \& Sielfeld 1976; Formas \& Pugín 1978; Ortiz \& Iturra 2011; Correa et al. 2011; Ortiz 2015).

Nannophryne variegata Günther, 1870 es una especie endémica de los bosques australes de Chile y Argentina (Boulenger 1882; Cope 1890; Philippi 1902; Cei 1962) (Fig. 1a). Se caracteriza por ser uno de los anfibios con distribución más austral del mundo junto a $B$. antartandica Barrio, 1967 (Úbeda et al. 2010). En Chile se distribuye desde la Región de la Araucanía hasta la Región de Magallanes y de la Antártica Chilena (Cei 1962). En Argentina ha sido descrito para las provincias de Neuquén, Río Negro, Chubut y Santa Cruz (Berg 1897; Basso \& Williams 1997; Úbeda 1998) y su distribución comprende las regiones ecológicas: Andina, Oceánica de influencia mediterránea, Oceánica temperada fría y Oceánica subantártica (Veloso \& Navarro 1988). Estas últimas regiones se caracterizan por tener temperaturas medias anuales bajas, con constante precipitación y fuertes vientos (Hock 1967).

Esta especie es abundante localmente y generalista en la selección de hábitat (Díaz-Páez \& Ortiz 2003; Úbeda et al. 2010) con una tendencia ecológica hacia los pequeños cuerpos de aguas someros y temporales (Formas 1981) y ha sido descrita como una especie típica de áreas preandinas y andinas cubiertas por bosque de Nothofagus ( $N$. antarctica, N. dombeyi, N. pumilio) (Formas \& Pugín 1978; Rabanal \& Nuñez 2009). En el sector norte de su distribución, Capurro (1957) la encontró bajo troncos caídos en proceso de putrefacción y entre los arbustos que quedan en medio de los bosques de Araucaria imbricata (= A. araucana) que trepan sobre las primeras estribaciones de los Andes. En el sector sur de los bosques más australes es común encontrarla en montículos de musgos o bajo piedras y troncos caídos, frecuentemente asociada a la comunidad de turberas y tundra magallánica (Fig. 1b, 1c) (Díaz-Páez et al. 2002; Ortiz 2015).

En el extremo sur de la distribución en Chile, han sido descritos muy pocos registros debido a que existen extensos territorios continentales e insulares inaccesibles y aún inexplorados (Díaz Páez et al. 2002; Díaz \& Young 2004; Ortiz \& Iturra 2011; Ortiz 2015). Pese a esto, la especie ha sido reportada a través de informes técnicos en la Reserva Nacional Lago Cochrane (= Tamango) (Saucedo 2004), Reserva Nacional Las Guaitecas (Díaz-Páez et al. 2002), Reserva Nacional Katalalixar (Díaz \& Young 2004) y cuencas de los ríos Baker y Pascua (Ortiz et al. 2007). El registro más austral en Chile corresponde a Isthmus Bay, en la provincia de Última Esperanza (52 $10^{\prime} 46^{\prime \prime}$; 7340'30") (Ohlin 1896) y en Argentina en las costas del Lago del Desierto y Río de las Vueltas (Basso \& Williams 1997).

El objetivo de este trabajo es informar sobre nuevos registros de distribución de $N$. variegata en el sur de Chile y Argentina y compilar todos los registros reportados en la literatura y Museos, con la finalidad de describir su real rango de distribución, las características de los hábitats que ocupa y la representación en áreas protegidas de ambos países.

\section{METODOLOGÍA}

Para la recopilación de las localidades donde ha sido encontrada $N$. variegata, se consideraron los registros de las colecciones herpetológicas del Museo de Zoología de la Universidad de Concepción (MZUC) (los que corresponden a los nuevos registros), Field Museum of Natural History (FMNH), Ex Centro Nacional de Investigaciones Iológicas (Ex CENAI, actualmente en MACN), Fundación Miguel Lillo (FML), Museo Argentino de Ciencias Naturales (MACN), Museo de La Plata (MLP) y de la Colección Plan Inventario (Parques Nacionales Lanín y Nahuel Huapi) para la Administración de Parques Nacionales, Bariloche (PI), las que fueron complementadas con aquellas localidades obtenidas de la literatura y con observaciones personales de los autores (TABLA 1). Para la determinación de las coordenadas geográficas de las localidades obtenidas de la literatura se realizó una aproximación a los posibles lugares señalados en el texto. Para las localidades de Museos se utilizaron las coordenadas indicadas en las fichas de recolección. Las nuevas localidades aportadas por los autores fueron calculadas con GPS en el terreno.

Las coordenadas geográficas fueron proyectadas sobre un mapa mediante el programa Arcmap (ESRI 2011; ArcGIS Desktop: Release 10. Redlands CA: Environmental Systems Research Institute) (Fig. 2). Además, se construyó un mapa con las áreas silvestres protegidas del estado de Chile (SNASPE) y los parques nacionales de Argentina, con la finalidad de visualizar la representatividad de su distribución en estas áreas (Fig. 3). Las coberturas fueron descargadas desde http://www.ide.cl para Chile y obtenidas desde la Dirección Regional Patagonia Norte de la Administración de Parques Nacionales para Argentina.

\section{RESULTADOS}

\section{DisTRIBUCIÓN}

Nannophryne variegata se distribuye en el sur de Chile y Argentina, a lo largo de una extensa franja del bosque templado austral a ambos lados de la cordillera de los Andes. A lo largo de este amplio rango latitudinal de distribución, $N$. variegata habita diferentes ambientes que comprenden asociaciones boscosas húmedas (Fig. 1b), turberas, tundra magallánica (Fig. 1c y 1d) y ambientes altoandinos vecinos. Estos registros abarcan un rango latitudinal de distribución desde $37^{\circ} 28^{\prime}$ hasta los $52^{\circ} 10^{\prime}$ latitud Sur (Fig. 2).

En la vertiente occidental de la cordillera de los Andes, en territorio chileno, $N$. variegata en su distribución norte se 
encuentra en asociaciones boscosas de robles (Nothofagus), araucarias (Araucaria) y humedales montanos (mallines) rodeados de bosque de alerce (Fitzroya cupressoides). En el sur prefiere matorrales con predominio de ciprés de las Guaitecas (Pilgerodendron uviferum) o turberas y tundra magallánica. En la vertiente oriental de la cordillera de los Andes, en territorio argentino, habita distintos tipos de asociaciones boscosas dominadas por especies de Nothofagus como coihue ( $N$. dombeyi), raulí ( $N$. alpina), roble pellín (N. oblicua) y coihue de Magallanes $(N$. betuloides), que pueden contener otras especies arbóreas como araucaria y alerce.

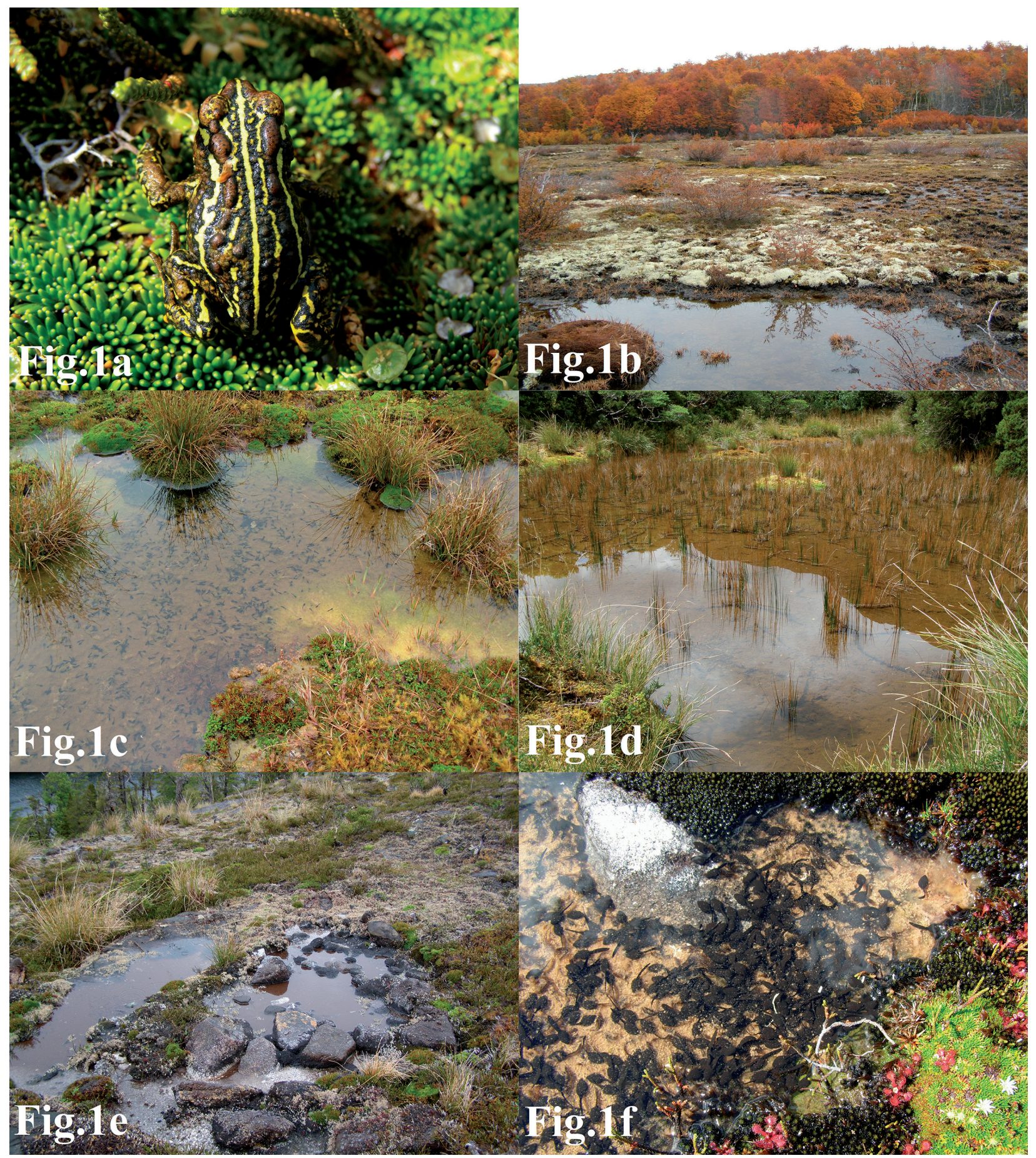

Figura 1. Nannophryne variegata y algunos hábitats donde se encuentra. 1a Ejemplar adulto; 1b Bosque de Nothofagus; 1c, 1d y 1e Turberas y tundra magallánica; 1f Pozón con larvas. / Nannophryne variegata and its habitats. 1a Adult specimen; 1b Nothofagus Forest; 1c, 1d and 1e Magellan tundra and peat bogs; $1 \mathrm{f}$ Pool with larvae. 
Las poblaciones más orientales de la especie se encuentran en territorio argentino, a partir de los $71^{\circ}$ Oeste, en el límite oriental del bosque de lenga ( $N$. pumilio), principalmente en los Parques Nacionales Lanín y Nahuel Huapi y las poblaciones más occidentales se encuentran en territorio chileno hasta los $75^{\circ}$ de longitud Oeste en Isla Esmeralda, provincia de Última Esperanza, caracterizada por poseer una vegetación de tipo turbal.

En cuanto a la distribución altitudinal, la especie se distribuye desde el nivel del mar hasta los 2200 m s.n.m., abarcando diferentes pisos altitudinales de bosque y humedales altoandinos por encima y próximos al límite superior del bosque de lenga.

\section{HÁBITATS}

Dentro de la variedad de ambientes boscosos, la especie utiliza como hábitats reproductivos aguas quietas (o con muy poca corriente), someras y soleadas, tales como charcas, pequeñas lagunas, pozas dentro de turberas y mallines y humedales altoandinos (Fig. 1e). Además, puede utilizar sectores de aguas quietas en nacientes de arroyos de montaña. La reproducción se realiza en las pozas que quedan entre los cojines de Sphagnum, en pozones presentes sobre sustratos rocosos rodeados de turba o en áreas anegadas formadas por el derretimiento de la nieve en ambientes de alta montaña (Fig. 1f).

En sus hábitats reproductivos, los renacuajos de $N$. variegata pueden ser sintópicos con los de $B$. antartandica (Ortiz obs. pers.) y los de Pleurodema thaul (Formas \& Pugín 1978). En el sur de su distribución pueden cohabitar con los renacuajos de Ch. grandisonae.

\section{REPRESENTATIVIDAD EN ÁREAS PROTEGIDAS}

El área de distribución de $N$. variegata está bien representada en áreas silvestres protegidas del estado (SNASPE) en Chile y en parques y reservas nacionales y otras áreas naturales protegidas de Argentina (TABLA 1 y Fig. 3). En Chile, $N$. variegata se encuentra en seis Parques Nacionales (Laguna del Laja, Los Paraguas (=Conguillío), Vicente Pérez Rosales, Alerce Andino, Laguna San Rafael y Bernardo O'Higgins), además, posiblemente en Torres del Paine (Markham 1971) y en tres Reservas Nacionales (Las Guaitecas, Lago Cochrane (= Tamango) y Katalalixar). En territorio argentino, $N$. variegata se ha reportado en los Parques Nacionales Lanín, Nahuel Huapi, Los Alerces, Perito Moreno y Los Glaciares y en el Parque Provincial Copahue.

\section{DISCUSIÓN}

La especie fue descrita por Günther (1870) a partir de ejemplares recolectados en tres localidades del oeste de la Patagonia, Puerto Bueno $\left(51^{\circ} 00^{\prime} \mathrm{S} ; 7^{\circ} 18^{\prime} \mathrm{O}\right)$,
Puerto Grappler $\left(49^{\circ} 24^{\prime} \mathrm{S} ; 7^{\circ} 19^{\prime} \mathrm{O}\right)$ y Puerto Edén (4909'S; 74²7’O), pero según Cei (1962), su terra típica correspondería a Puerto Bueno (Magallanes, Chile). Donoso-Barros (1974) indicó que en territorio chileno la localidad más septentrional de $N$. variegata sería la Sierra Velluda, sector Los Barros en el Parque Nacional Laguna del Laja. Sin embargo, esto no ha podido ser corroborado por diversos investigadores que han inspeccionado dicha área. Formas \& Pugín (1978) mencionan que la localidad más al norte encontrada en Chile correspondería a las cercanías del volcán Llaima (Provincia de Cautín) (3841'S; $71^{\circ} 43^{\prime}$ O) donde la especie estaría asociada a una laguna permanente, que se cubre con nieve durante el invierno y donde dominan $N$. pumilio, A. araucana, Maytenus distichia (chaurilla), Drimys winteri (canelo), Ribes sp., Agrostis sp. y Juncus procerus (junquillo). Registros posteriores confirman su presencia en el Parque Nacional Los Paraguas (= Conguillío) (3840’S; 71³9’O) (Pugín \& Garrido 1981) y en las cercanías de Liucura, próximo a la Reserva Nacional Alto Biobío (Ortiz, obs. pers.) (3845’S; $70^{\circ} 58^{\prime}$ O). Sin embargo, en la colección del Field Museum of Natural History de Chicago se encuentran ejemplares recolectados al norte de estas localidades, en la cordillera de Pemehue ( $\left.38^{\circ} 09^{\prime} \mathrm{S} ; 7^{\circ} 40^{\prime} \mathrm{O}\right)$ y en Termas de Tolhuaca (38 $\left.14^{\circ} \mathrm{S} ; 71^{\circ} 43^{\prime} \mathrm{O}\right)$.

Por otra parte, el registro publicado más austral de presencia de esta especie corresponde a la localidad de Isthmus Bay $\left(52^{\circ} 10^{\prime} \mathrm{S} ; 7^{\circ} 40^{\prime} \mathrm{O}\right)$ en la provincia de Última Esperanza, Chile (Ohlin 1896). De esta manera $N$. variegata, junto con las especies simpátricas $B$. antartandica, reportada hasta los $51^{\circ} 31^{\prime} \mathrm{S}, 7^{\circ} 55^{\prime} \mathrm{O}$ en Isla Virtudes, Región de Magallanes (Atalah \& Sielfeld 1976), $P$.

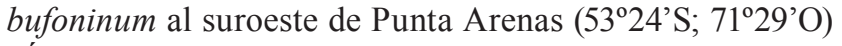
(Úbeda et al. 2010) y Ch. grandisonae en el Parque Marino Francisco Coloane (53⒉'S; $\left.72^{\circ} 37^{\prime} \mathrm{O}\right)$ (Ortiz 2015), son las especies de anuros más australes de Chile y del mundo.

Con respecto a su representatividad en Argentina, sus límites de distribución estarían dados por el Parque Provincial Copahue $\left(37^{\circ} 51^{\prime} \mathrm{S} ; 71^{\circ} 04^{\prime} \mathrm{O}\right)$ en la provincia de Neuquén (Martín 1988) en el norte, mientras que, en el sur, por la localidad de Chorrillo del Salto (49 $17^{\prime} 57^{\prime \prime}$ S; 72 54'14" O) en el Parque Nacional Glaciares, provincia de Santa Cruz (Corvalán 2011). Los nuevos registros de distribución oriental corresponden al Parque Nacional Nahuel Huapi y al área de Lago del Desierto (C. Úbeda, obs. pers.) (TABLA 1).

Como se observa en la Figura 2, N. variegata no presenta distribución continua, con la existencia de un vacío de información entre las latitudes de $42^{\circ}$ y $45^{\circ} \mathrm{S}$. Esta discontinuidad de registros por el lado occidental puede deberse a falta de exploración en las provincias de Palena, Coyhaique y General Carrera, las que se caracterizan por tener un clima templado lluvioso con temperaturas moderadas a bajas sin grandes variaciones a lo largo del 
año (di Castri \& Hajek 1976), lo que permitiría la presencia de esta especie. Además, se debe considerar que las características del paisaje han sido modeladas a través de los cambios climáticos y geomorfológicos ocurridos durante fines del Terciario y comienzos del Cuaternario (Dollenz 1995; Thorson 1999; McCulloch et al. 2000; Villagrán et al. 2004). Las glaciaciones cíclicas ocurridas en el pasado (Rabassa et al. 2005) han modelado el escenario actual, donde los recurrentes avances y retrocesos de los glaciares provocaron el descenso de la temperatura y un aumento de precipitaciones (Pisano 1997), generando condiciones que permiten suponer un aumento en la disponibilidad de hábitat. Esta situación supondría que $N$. variegata se encontraría en áreas aún poco exploradas que fueron directamente afectadas por las glaciaciones.

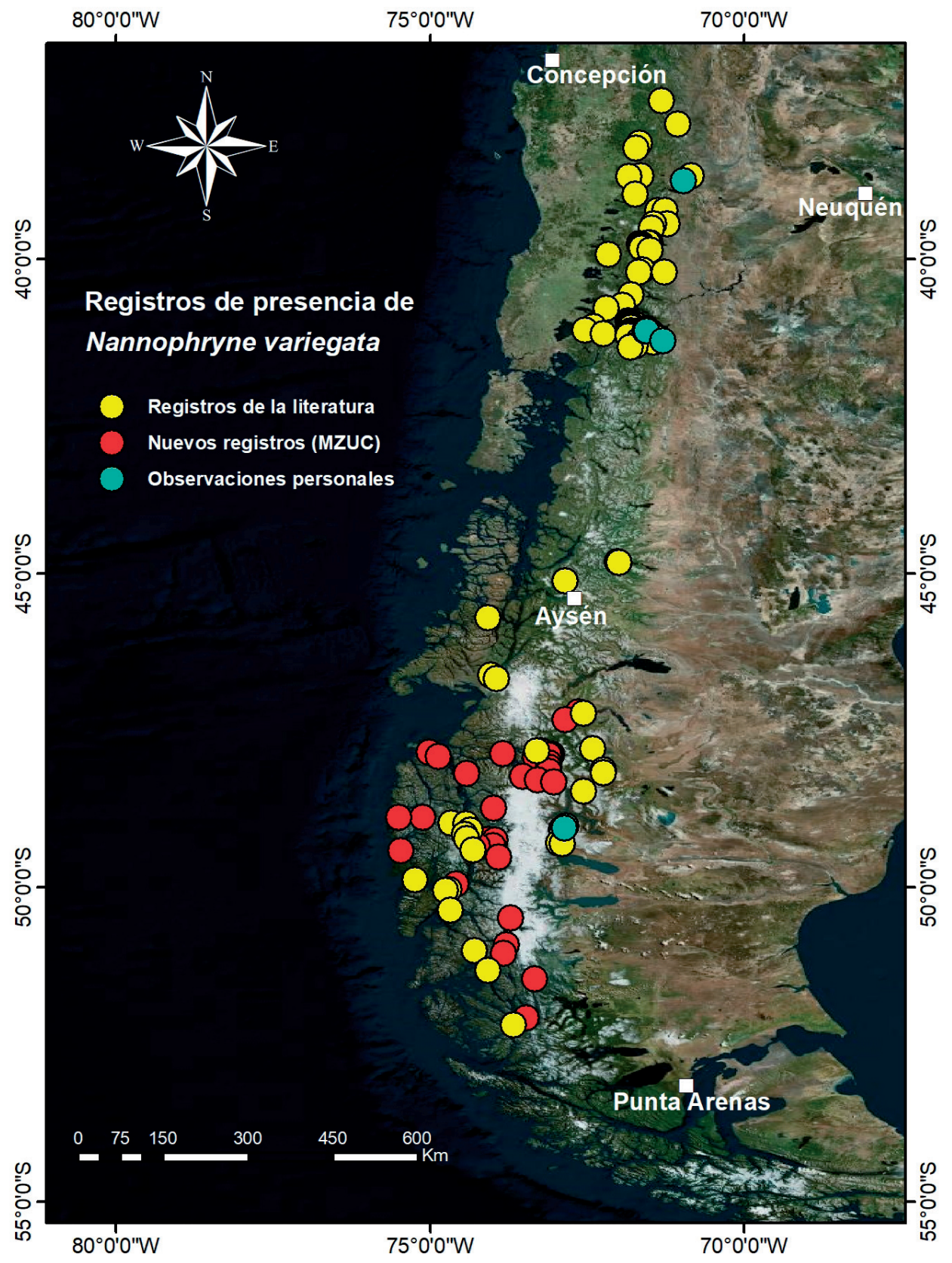

Figura 2. Distribución geográfica de Nannophryne variegata en Chile y Argentina. Los círculos amarillos representan las localidades obtenidas desde la literatura y ejemplares de Museos. Los círculos rojos representan nuevas localidades muestreadas en Chile; los círculos celestes representan observaciones personales de los autores. / Geographical distribution of Nannophryne variegata in Chile and Argentina. The yellow circle represents localities obtained from literature and Museum samples. The red circles represent new localities sampled in Chile; the light blue circles represent personal observations of the authors. 
Por la amplia distribución latitudinal que presenta la especie, así como por el gran número de poblaciones observadas, $N$. variegata ha sido categorizada como especie de Preocupación Menor tanto por el Ministerio del Medio Ambiente de Chile (D.S. no 41 del año 2011) como por la IUCN (Úbeda et al. 2010). En Argentina se ha categorizado como No Amenazada (Resolución 1055/13, Ex Secretaría de
Ambiente Humano y Desarrollo Sustentable). De acuerdo a estos nuevos registros, se considera mantener esta categoría de conservación debido a que, de un total de 132 registros, más de 75 se encuentran dentro de áreas protegidas, a lo que se debe agregar la lejanía de su distribución de centros urbanos.

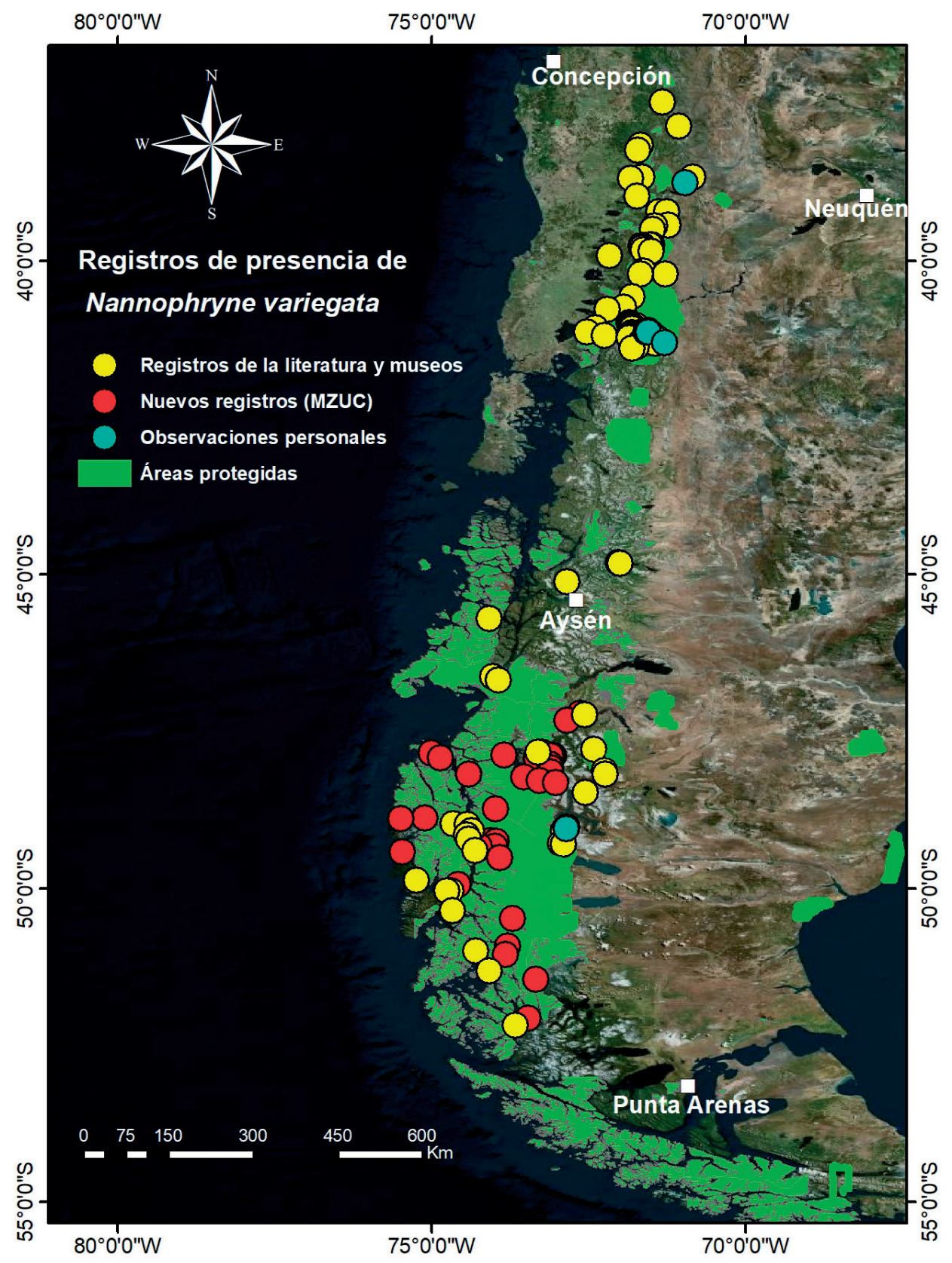

Figura 3. Representatividad de Nannophryne variegata en las áreas protegidas de Chile (SNASPE) y parques nacionales de Argentina. La superficie verde representa las áreas protegidas en Chile y parques nacionales en Argentina. / Representation of the distribution of Nannophryne variegata in protected areas of Chile and Argentina. The green surface represents the protected areas in Chile and the national parks in Argentina. 


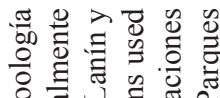
s

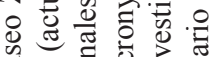

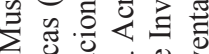

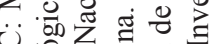

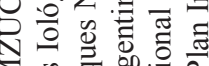
年

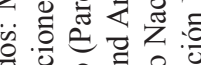

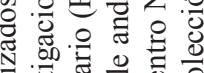

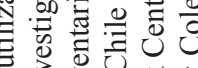

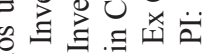

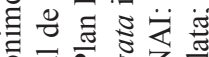

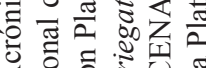
(4)

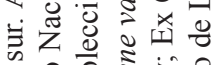

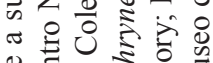

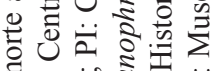

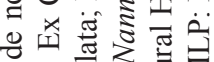

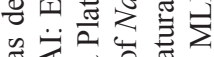

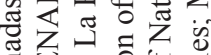

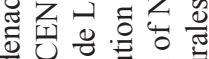

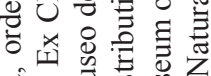

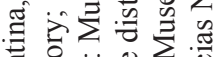

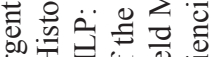

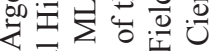

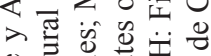

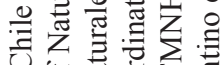
5응

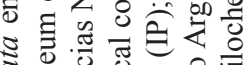

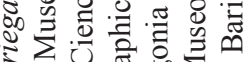

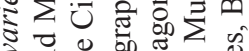

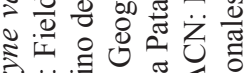

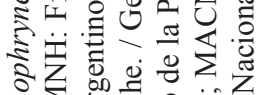

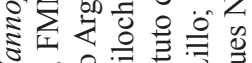

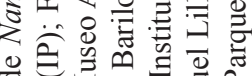
. . 드의. 항 哓 으을

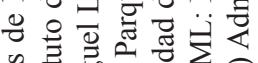

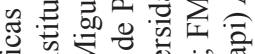

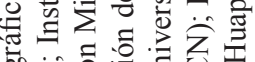
额, 吅:

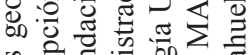

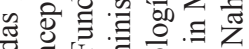

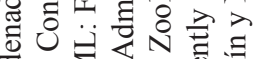

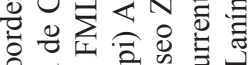

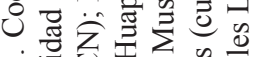

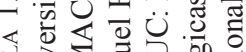

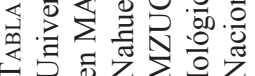

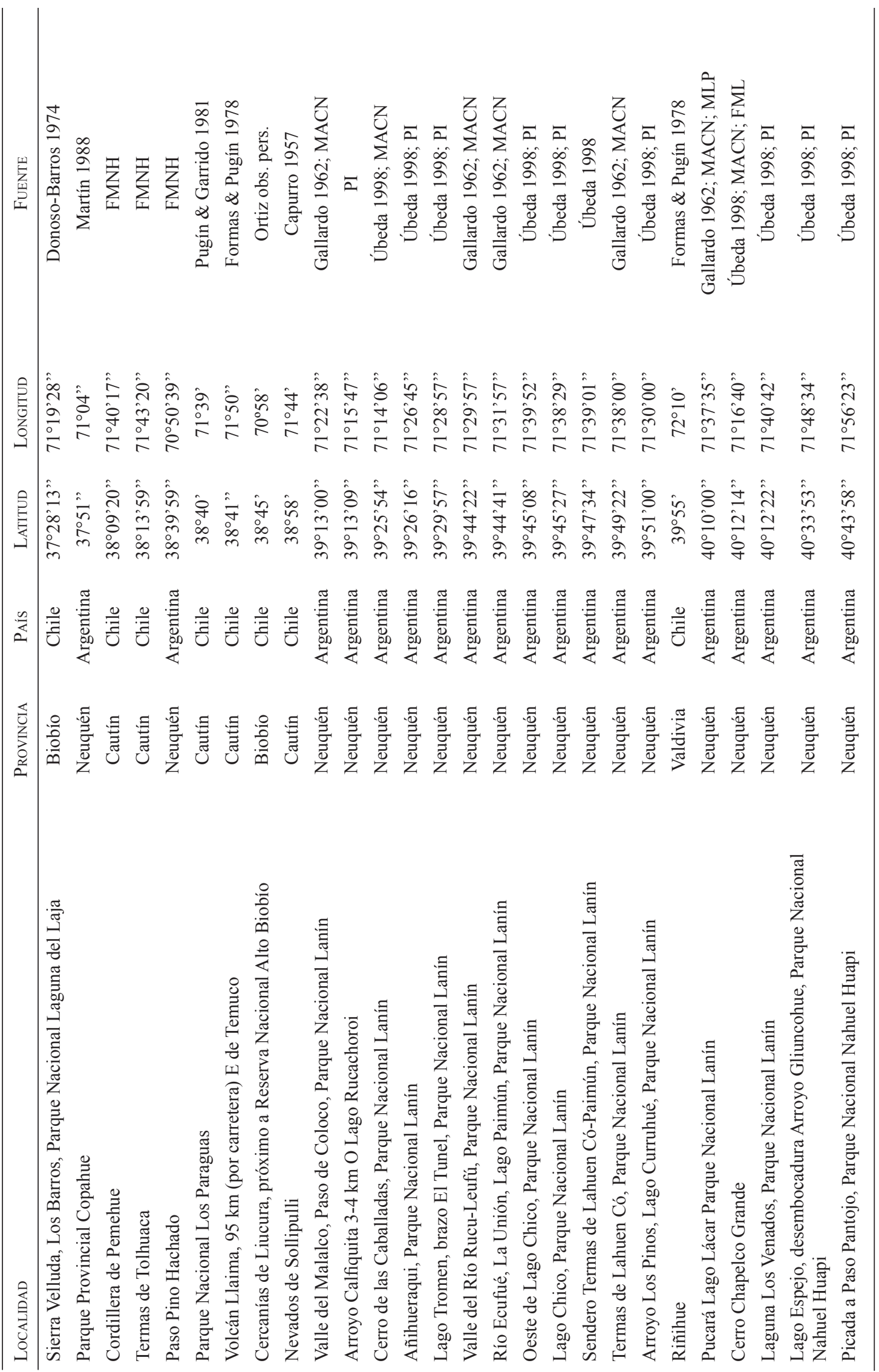




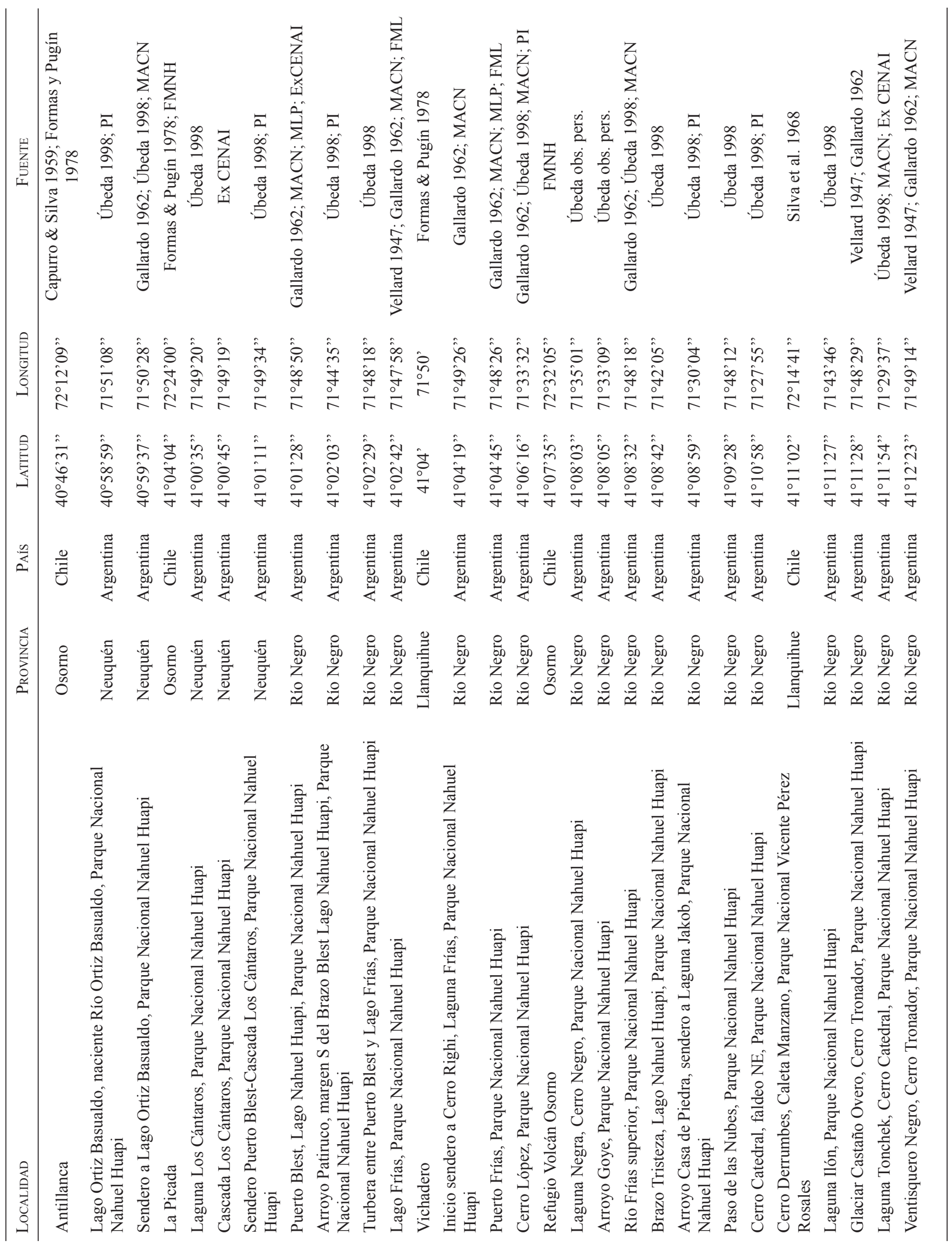




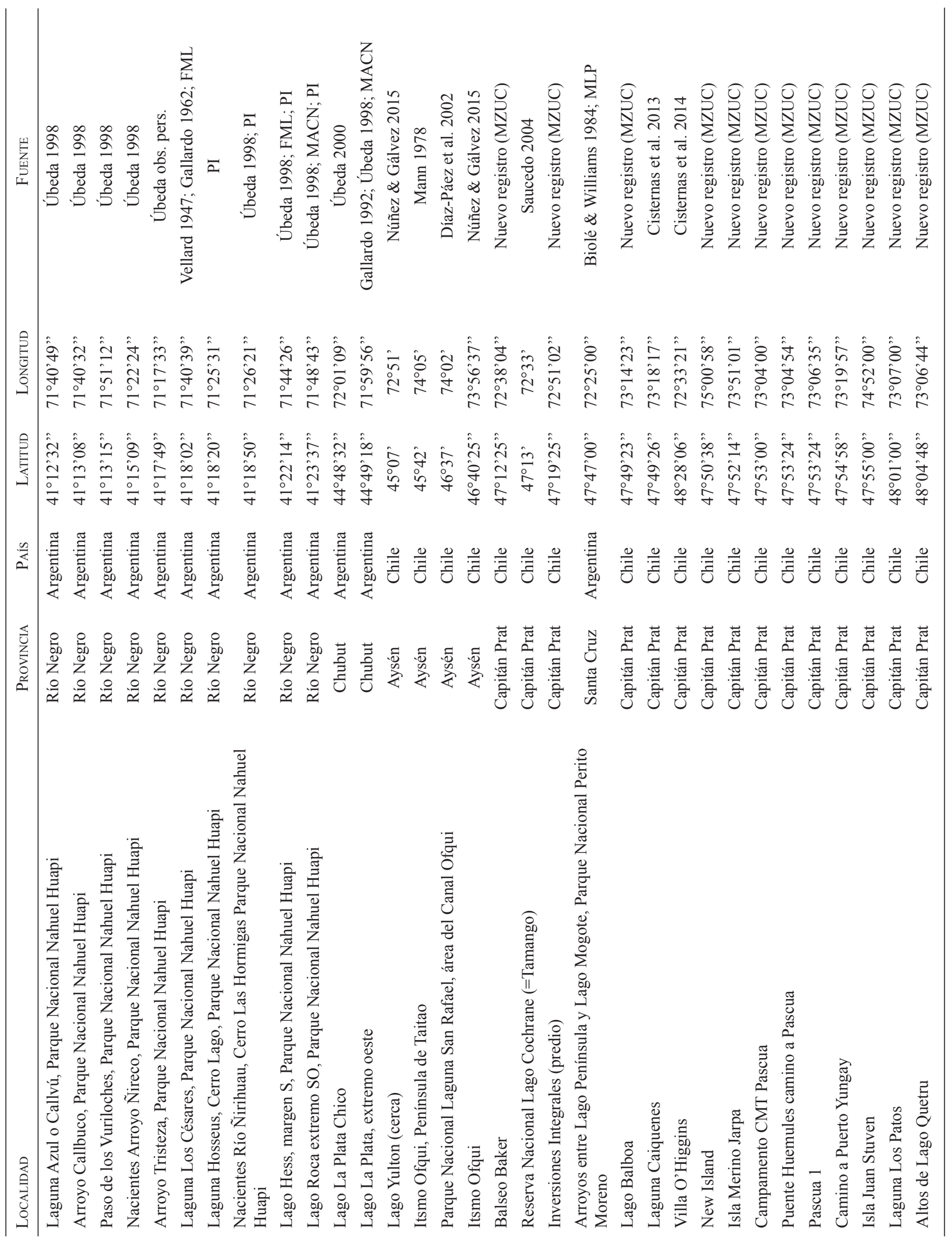




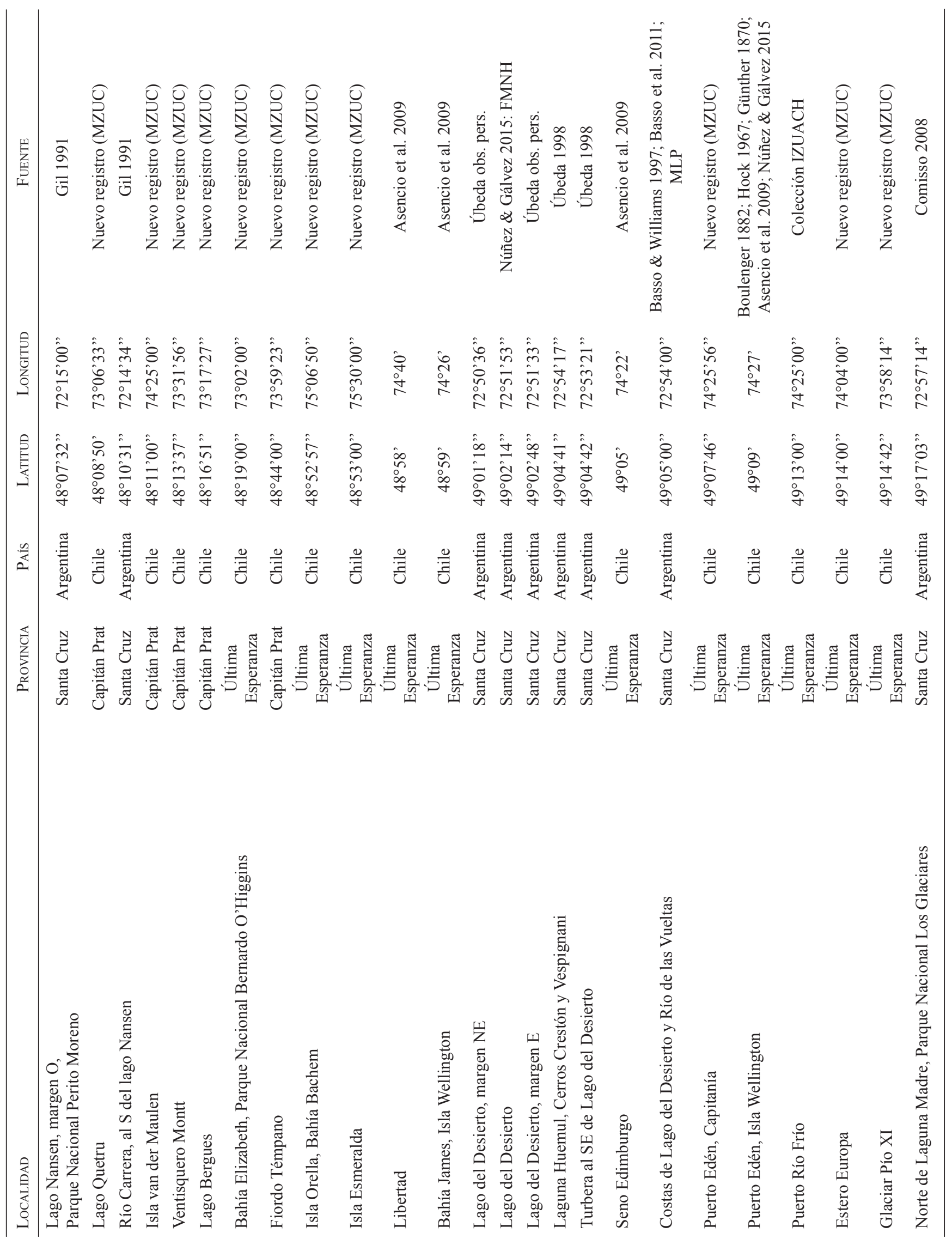


Distribución geográfica de Nannophryne variegata: Cisternas-Medina, I. ET AL.

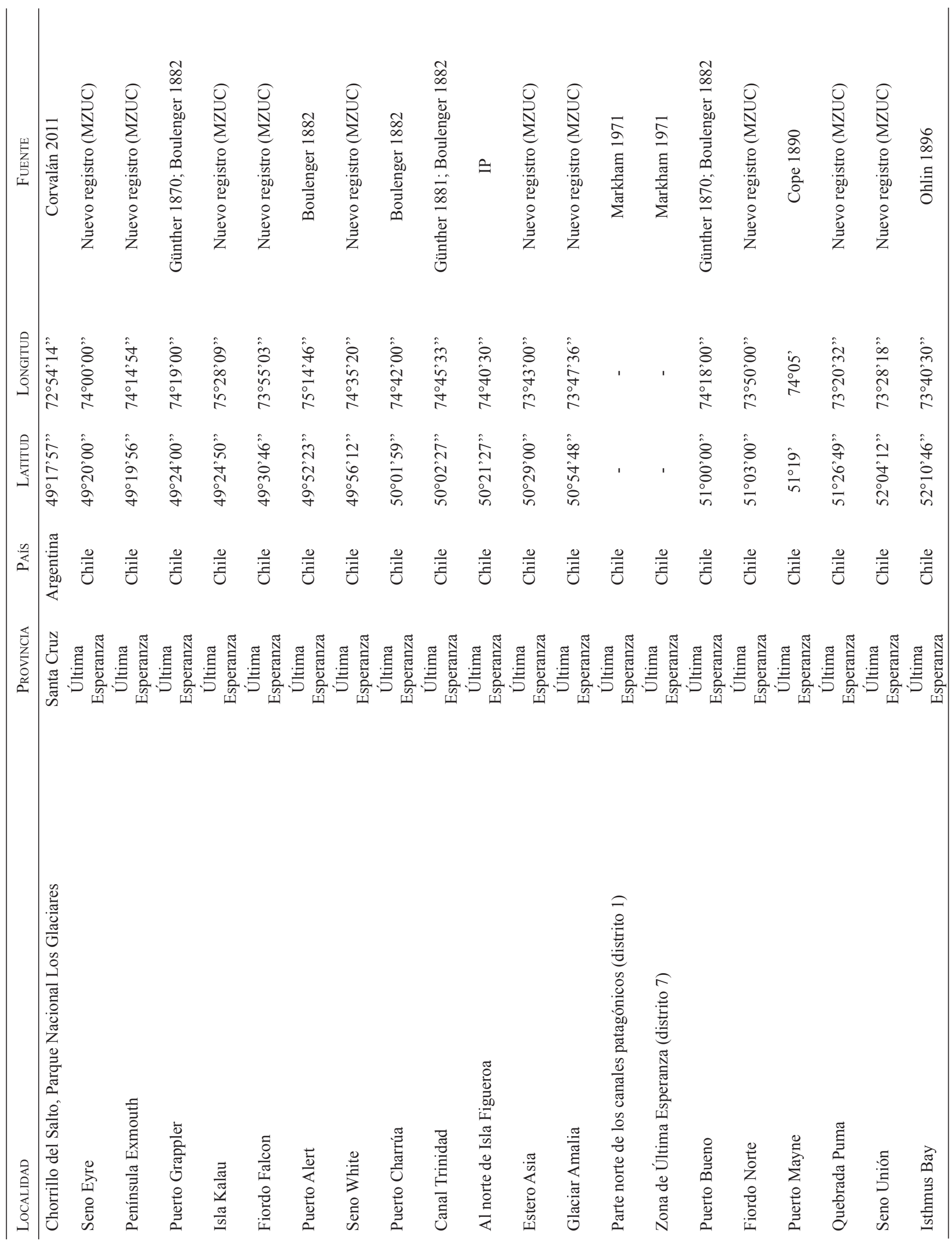




\section{AGRADECIMIENTOS}

Este trabajo se financió en parte con los proyectos de "Determinación de la línea base de fauna terrestre en la zona sur de la XI Región de Aysén relacionada con el proyecto hidroeléctrico Aysén y sus respectivos estudios complementarios" de la Vicerrectoría de Investigación y Desarrollo de la Universidad de Concepción, así como el de "Caracterización territorial del Parque Nacional Bernardo O'Higgins: Su potencial económico, turístico, científico y cultural" INNOVA-CORFO 08CTU01-20, Fundación CEQUA. Se agradece al Servicio Agrícola y Ganadero por las resoluciones exentas $n^{\circ} 2160,6491,29$ y 1740 de los años 2006, 2007, 2008 y 2009 respectivamente, a Ximena Silva del Instituto de la Patagonia y a los revisores que realizaron comentarios que mejoraron la primera versión de este trabajo.

\section{REFERENCIAS}

Asencio, J., Kusch, A., Henríquez, J.M., Cárcamo, J. 2009. Registro de Anfibios en el bosque Norpatagónico costero de Canal Messier, Chile. Anales del Instituto de la Patagonia (Chile) 37: 113-116.

Atalah, A., Sielfeld, W. 1976. Presencia de Batrachyla antartandica Barrio en Magallanes. Anales del Instituto de la Patagonia (Chile) 7: 168-170.

BARrio, A. 1967. Batrachyla antartandica n. sp. (Anura: Leptodactylidae). Descripción y estudio comparativo con la especie genotípica, B. leptopus Bell. Physis (Buenos Aires) 27(74): 101-109.

Basso, N. G., Williams, J. D. 1997. Bufo variegatus (Eden Harbour Toad). Herpetological Review 28(2): 92.

Basso, N.G., Úbeda, C.A., Bunge, M.M., Martinazzo, L.B. 2011. A new genus of neobatrachian frog from southern Patagonian forests, Argentina and Chile. Zootaxa 3002: 31-44.

Berg, C. 1897. Batracios argentinos. Enumeración sistemática, sinonímica y bibliográfica de los batracios de la República Argentina. Anales del Museo Nacional de Buenos Aires 5(2): 147-226.

Biolé, F., Williams, J.D. 1984. Nueva localidad para Bufo variegatus (Günther, 1870) (Amphibia: Anura) y notas preliminares sobre su variación geográfica. Neotropica 30(84): 243-244.

Boulenger, G.A. 1882. Catalogue of the Batrachia Salientia s. Ecaudata in the collection of the British Museum. $2 \mathrm{~d}$ ed.

CAPurro, L. 1957. Anfibios de la región de los lagos valdivianos. Investigaciones Zoológicas Chilenas 4(1): 21-28.

Capurro, L., Silva, F. 1959. Valor taxonómico del estudio cromatográfico del veneno de las parótidas de Bufo spinulosus y Bufo variegatus. Investigaciones Zoológicas Chilenas 5: 189-197.

CEI, J.M. 1962. Batracios de Chile. Ediciones Universidad de Chile, Santiago. CVIII + 128 pp.

Cisternas, J., Correa, C., Velásquez, N., Penna, M. 2013. Reproductive features of Chaltenobatrachus grandisonae
(Anura: Batrachylidae) within a protected area in Patagonia, Chile. Revista Chilena de Historia Natural 86: 365-368.

Cisternas, J., Correa, C., Lopez, L., Riveros, Y., Silva, C. 2014. Origen y desarrollo del proyecto "Estudio de los Anfibios de Villa O'Higgins": bitácora de cuatro años de trabajo. Boletín Chileno de Herpetología 1: 5-7.

Comisso, S.E. 2008. Primera Cita de Nannophryne variegata en el Parque Nacional Los Glaciares. En Administración de Parques Nacionales. Sistema de Información de Biodiversidad. URL: http://www.sib.gov.ar

Cope, E.D. 1890. Scientific result of exploration by the US. Fish Commission Steamer Albatross. No. III Report on the batrachians and reptiles collected in 1887-88. Proceeding of the United States National Museum 12: 141-147.

Correa, C., Cisternas, J., Correa-Solís, M. 2011. Lista comentada de las especies de anfibios de Chile (Amphibia: Anura). Boletín de Biodiversidad de Chile 6: 1-21.

Corvalán, C. 2011. Informe de registro ocasional de sapito de tres rayas (Nannophryne variegata) en el PN Los Glaciares. En: Administración de Parques Nacionales. Sistema de Información de Biodiversidad. URL: http://www.sib.gov.ar

Di castri, F., Hajek, E. 1976. Bioclimatología de Chile. Ediciones Universidad Católica de Chile. Lira 140 Santiago, Chile. $128 \mathrm{pp}$.

Díaz, H., Young, S. 2004. Diversidad de Anfibios en Aysén. En: Howorth, R. (ed.). Resúmenes: líneas de investigaciones científicas. Proyecto biodiversidad de Aysén. Manejo sustentable de áreas protegidas región Aysén. 2 pp.

DíAz-PÁez, H., Ortiz, J.C. 2003. Evaluación del estado de conservación de los anfibios en Chile. Revista Chilena de Historia Natural 76: 509-525.

Díaz-PÁez, H., Williams, C., Griffiths, R.A. 2002. Diversidad y abundancia de anfibios en el Parque Nacional "Laguna San Rafael” (XI Región, Chile). Boletín del Museo Nacional de Historia Natural (Chile) 51: 135-145.

Dollenz, A. 1995. Los árboles y bosques de Magallanes. Ediciones Universidad de Magallanes, Punta Arenas, Chile. 32pp.

Donoso-Barros, R. 1974. Notas Herpetológicas. Boletín de la Sociedad de Biología de Concepción (Chile) 47: 285-287.

Formas, J.R. 1981. Adaptaciones larvarias de los anuros del bosque temperado Austral de Sudamérica. Medio Ambiente 5: 15-21.

Formas, J. R. 1979. La herpetofauna de los bosques temperados de Sudamérica. En: Duellman, W. E. (ed) The South American Herpetofauna: Its Origen, Evolution, and Dispersal: 341379. Museum of Natural History, University of Kansas, Monograph 7, Lawrence, Kansas.

Formas, J.R., Pugín, E. 1978. Tadpoles of Eupsophus roseus and Bufo variegatus (Amphibia, Anura) in Southern Chile. Journal of Herpetology 12(2): 243-246.

Gallardo, J.M. 1962. A propósito de Bufo variegatus (Günther), sapo del bosque húmedo antartándico, y las otras especies de Bufo neotropicales. Physis (Buenos Aires) 23(64): 93102.

Gallardo, J.M. 1992. Una nueva localidad para Bufo variegatus (Günther) (Anura, Bufonidae), Lago La Plata, Chubut, Argentina. Boletín de la Asociación Herpetológica Argentina 8(2): 2-3

GIL, G. 1991. Informe sobre las observaciones complementarias 
realizadas durante la campaña '91 del Proyecto Huemul al P.N. Perito Moreno. Administración de Parques Nacionales, Argentina. 19 pp.

GÜNTHER, A. 1870. Second account of species of tailless Batrachian added to the collection of the British Museum. Proceeding of the Zoological Society, London. 402 pp.

Günther, A. 1881. Account of the Reptiles, Batrachians and Fishes collected during the Survey of H.M.S. "Alert" in the Straits of Magellan and the coast Patagonian. Proceeding of the Zoological Society, London 1881: 18-19.

Hock, R.J. 1967. Temperature effect on breeding of the toad, Bufo variegatus, in southern Chile. Copeia 1: 227-230.

ManN, G. 1978. Bufo variegatus (No common name). Chile. Herpetological Review 9(3): 107.

Markham, B. 1971. Catálogo de anfibios, reptiles, aves y mamíferos de la Provincia de Magallanes (Chile). Instituto de la Patagonia, Punta Arenas. 64pp.

Martín, C. 1988. Plan general de manejo del Parque Provincial Copahue. Dirección Provincial de Turismo, Neuquén, Argentina. 105 pp.

Mc Culloch, R.D., Bentley, M.J., Purves, R.S. 2000. Climatic inferences from glacial and palaeoecological evidence at the last glacial termination, southern South America. Journal of Quaternary Science 15: 409-417.

Ministerio del Medio Ambiente. 2011. D.S. no 41/ 2011. Diario oficial de la República de Chile, miércoles 11 de abril de 2012.

NúÑEz, H., GÁlvez, O. 2015. Catálogo de la colección herpetológica del Museo Nacional de Historia Natural y nomenclátor basado en la colección. Museo Nacional de Historia Natural. Publicación Ocasional 64: 1-203.

Ohlin, A. 1896. A zoologist in Tierra del Fuego. Some accounts of the Swedish Expedition 1895/1896. Natural Science 9: 172-181.

Ortiz, J.C. 2015. Anfibios de las turberas del extremo austral de Chile. Cap. 8 p. 227-238. En: Domínguez, E., VegaValdés, D. (eds.). Funciones y servicios ecosistémicos de las turberas en Magallanes. Colección de libros INIA $\mathrm{N}^{\mathrm{o}}$ 33.Instituto de Investigaciones Agropecuarias. Centro Regional de Investigación Kampenaike. Punta Arenas, Chile. 334 pp.

Ortiz, J.C., Iturra, M. 2011. Fauna terrestre, Anfibios: 84-87. En: CEQUA (ed.) Parque Nacional Bernardo O’Higgins. 248pp.

Ortiz, J.C., Jerez, V., Figueroa, R., Corales, S., Schlatter, R., Valenzuela, J., Victoriano, P., Díaz, H., Nuñez, J.J., Parra, L., Casanueva, M.E., Aguilera, D’elía, G.D., Guzmán., J., Sielfeld, W. 2007. Informe Final de la Determinación de la línea base de fauna terrestre en la zona sur de la XI Región de Aysén. Universidad de Concepción.

PhILIPPI, R.A. 1902. Suplemento a los Batraquios chilenos descritos en la Historia Física y Política de Chile de don Claudio Gay. Santiago, Chile 1902: 1-161.
PISANO, E. 1977. Fitogeografía de Fuego-Patagonia chilena. I. Comunidades vegetales entre la latitudes 52 y $56^{\circ} \mathrm{S}$. Anales Instituto de la Patagonia, Punta Arenas (Chile) 8:121-250.

Pugín, E., Garrido, O. 1981. Morfología espermática en algunas especies de anuros pertenecientes al bosque temperado del sur de Chile. Ultraestructura comparada. Medio Ambiente 5(1-2): 45-57.

Rabanal, F.E., NuÑEz, J.J. 2008 Anfibios de los bosques templados de Chile. Universidad Austral de Chile, Valdivia, Chile. 206pp.

Rabassa, J., Coronato, A., Salemme, M. 2005. Chronology of the late Cenozoic Patagonian glaciation and their correlation with biostratigraphic units of the Pampean Region (Argentina). Journal of South American Earth Science 20: 81-103.

Saucedo, C. 2004. Documento trabajo N 406. 2004. Plan de manejo reserva nacional Lago Cochrane (Tamango) y lotes aledaños. CONAF 2004.

Secretaría de Ambiente humano y desarRollo sustentable. 2013. Resolución 1055/13. Boletín oficial 12 de septiembre de 2013, Argentina.

Silva, F., Veloso, A., Solervicens, J., Ortiz, J.C. 1968. Investigaciones zoológicas en el Parque Nacional "Vicente Pérez Rosales" y zona de Pargua. Noticiario Mensual del Museo Nacional de Historia Natural de Santiago 148: 3-12.

Thorson, R.M. 1999. The glacial limit in South America and its rol in biogeography: Darwin's observations. Ciencia al Día 4: $1-15$.

ÚBEDA, C.A. 1998. Batracofauna de los bosques templados patagónicos: un enfoque ecobiogeográfico. $\mathrm{PhD}$ Thesis (unpublished), Facultad de Ciencias Exactas y Naturales, Universidad de Buenos Aires, Buenos Aires, [i - xv] + $354 \mathrm{pp}$.

ÚBedA, C.A. 2000. Geographic Distribution. Batrachyla antartandica. Herpetological Review 31(2): 109.

Úbeda, C., Veloso, A., NúÑez, H., Basso, B., Blotto, B. 2010. Nannophryne variegata. The IUCN Red List of Threatened Species 2010: e.T54790A11192107. URL: http://www. iucnredlist.org/details/54790/0. Accedido: March 15, 2018.

Vellard, J. 1947. Dos batracios interesantes en la región de Bariloche. Acta Zoológica Lilloana 4: 145-153.

Veloso, A., Navarro, J. 1988. Lista sistemática y distribución geográfica de anfibios y reptiles de Chile. Bolletino del Museo Regionale di Scienze Naturali, Torino 6(2): 481539.

Villagrán, C., Armesto, J., Hinojosa, L.F., Cuvertino, J., Pérez, C., Medina, C. 2004. El enigmático origen del bosque relicto de Fray Jorge. Ediciones Universidad de La Serena, La Serena, Chile 1: 3- 43.

Recibido: 09.05 .2018

Aceptado: 22.01.2019 